\title{
Degeneracy and inversion of band structure for Wigner crystals on a closed helix
}

\author{
A. V. Zampetaki, ${ }^{1}$ J. Stockhofe, ${ }^{1}$ and P. Schmelcher ${ }^{1,2}$ \\ ${ }^{1}$ Zentrum für Optische Quantentechnologien, Universität Hamburg, Luruper Chaussee 149, 22761 Hamburg, Germany \\ ${ }^{2}$ The Hamburg Centre for Ultrafast Imaging, Luruper Chaussee 149, 22761 Hamburg, Germany
}

(Received 18 September 2014; published 10 February 2015)

\begin{abstract}
Constraining long-range interacting particles to move on a curved manifold can drastically alter their effective interactions. As a prototype we explore the structure and vibrational dynamics of crystalline configurations formed on a closed helix. We show that the ground state undergoes a pitchfork bifurcation from a symmetric polygonic to a zigzag-like configuration with increasing radius of the helix. Remarkably, we find that, for a specific value of the helix radius, below the bifurcation point, the vibrational frequency spectrum collapses to a single frequency. This allows for an essentially independent small-amplitude motion of the individual particles and, consequently, localized excitations can propagate in time without significant spreading. Upon increasing the radius beyond the degeneracy point, the band structure is inverted, with the out-of-phase oscillation mode becoming lower in frequency than the mode corresponding to the center-of-mass motion.
\end{abstract}

DOI: 10.1103/PhysRevA.91.023409

PACS number(s): 37.10.Ty, 37.90.+j, 61.50.-f, 63.20.D-

Introduction. A key ingredient for the formation of complex systems out of fundamental building blocks such as atoms or ions is the two-body interaction potential. Depending on its short- or long-range character, the corresponding many-body systems show very diverse properties and structural features [1-4]. Generically, the strength of the two-body potential decreases with increasing distance between the interacting particles. This situation changes drastically if the particles are not allowed to explore complete space but are forced to move on a curved manifold while keeping their original interactions. Hence the particles can interact via the dynamically forbidden dimensions, while their motional degree of freedom is constrained to the manifold.

A prototype system of this character is the helix, having constant curvature and torsion. Indeed, several works [5-10] have demonstrated unique novel features arising with respect to the structural and dynamical properties of charged (or dipolar) particles on a helix. First, one encounters that longrange interacting particles effectively experience forces of oscillatory character on the helix. Consequently, stable boundstate configurations exist for equally charged, repulsively interacting particles and can even be tuned in number and strength $[5,8]$. The resulting potential landscape for more particles is topologically very complex, leading to configurations with increasingly distorted symmetries. In the same line it has been shown that a zero-temperature second-order liquid-gas transition occurs at a critical field for interacting dipoles [7], and cluster formation as well as crystallization are possible [10]. Rendering the helix locally inhomogeneous, the center of mass (c.m.) of the particles couples to their relative motions, allowing for dissociation of bound states or binding of particles out of the scattering continuum [9].

In view of the structural complexity of helical long-rangeinteracting many-particle systems the natural but intriguing question emerges of what is the dynamical response and, in particular, the vibrational structure of such systems. To address this question we explore the properties and dynamics of crystalline configurations formed by identical charged particles, so-called Wigner crystals [11], confined on a closed helix. We find that tuning the geometry induces a structural transition reminiscent of the transverse zigzag buckling in linear Wigner crystals [12]. Due to the one-dimensional (1D) constraint that restricts the allowed excitations, this transition is accompanied by an unconventional deformation of the corresponding dispersion relation: For finite systems, there is a regime of inverted dispersion, with the out-of-phase mode being lowest in frequency. Even more, the transition passes through a stage where the complete linearization spectrum is essentially degenerate, such that any low-amplitude mode is an eigenmode of the system. Notably, localized excitations do not transfer energy into the rest of the chain.

Toroidal helix. We consider a system of $N$ identical charged particles of mass $m_{0}$ interacting via the repulsive Coulomb interaction and confined to move on a 1D toroidal helix [see Fig. 1(a)] parametrized as

$$
\mathbf{r}(u)=\left(\begin{array}{c}
{[R+r \cos (u)] \cos (a u)} \\
{[R+r \cos (u)] \sin (a u)} \\
r \sin (u)
\end{array}\right), \quad u \in[0,2 M \pi],
$$

with $R$ being the major radius of the torus, $r$ denoting the radius of the helix (minor radius of the torus), and $h$ referring to the helix pitch. The parameter $a=\frac{1}{M}$ is the inverse number of windings $M=\frac{2 \pi R}{h}$. The effective Coulomb potential resulting from the confinement reads

$$
V\left(u_{1}, u_{2}, \ldots u_{N}\right)=\frac{1}{2} \sum_{i, j=1, i \neq j}^{N} \frac{\lambda}{\left|\mathbf{r}\left(u_{i}\right)-\mathbf{r}\left(u_{j}\right)\right|},
$$

with $\lambda$ being the coupling constant.

We perform a scaling transformation [9], measuring distances in units of $2 h / \pi$ and time in units of $\left(\frac{8 m_{0} h^{3}}{\lambda \pi^{3}}\right)^{1 / 2}$. This results in $\lambda$ and $m_{0}$ being set to 1 in the following, while the dimensionless helix pitch is set to $h=\pi / 2$. Note that the c.m. degree of freedom is coupled to the relative coordinates $\Delta_{i}=u_{i+1}-u_{i}$ for $r \neq 0$ since the confining manifold is not a homogeneous helix [9]. For $r=0$ the toroidal helix degenerates to a ring of radius $R$ where c.m. separation holds. In this ring limit, there is a single stationary state; namely, the totally symmetric polygonic configuration. In contrast, the potential landscape of charged particles confined on helical 
(a)

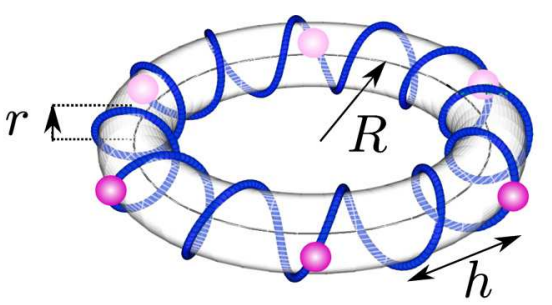

(b)

(c)

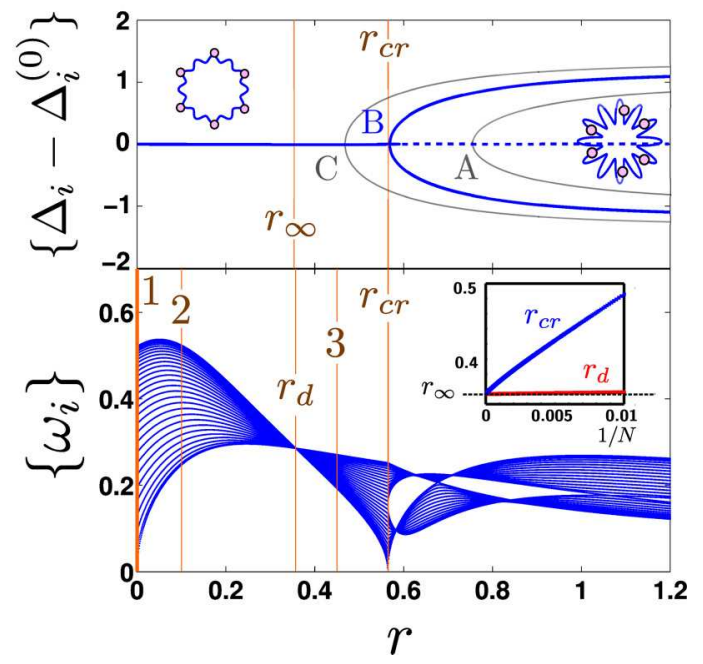

FIG. 1. (Color online) (a) Equidistant configuration of ions confined on the toroidal helix for $v=\frac{1}{2}$ and $N=6$. (b) Equilibrium displacements of particles in stationary configurations as a function of helix radius $r$ for filling $v=\frac{1}{2}$ and different numbers of particles: (A) $N=30$, (B) $N=60$, (C) $N=120$. All values of $\Delta_{i}-\Delta_{i}^{(0)}$ are plotted on top of each other: For the polygonic configuration, all nearest-neighbor interparticle distances are identical, $\Delta_{i}=\Delta_{i}^{(0)}$ for all $i$, while in the zigzag-like configuration precisely two different distances are found; see the insets. The vertical lines indicate $r_{\mathrm{cr}}$ for $N=60$ and the limiting value $r_{\infty}$ of $r_{\mathrm{cr}}$ in the thermodynamic limit. (c) Linearization spectrum as a function of $r$ of the stable solution (polygonic for $r<r_{\mathrm{cr}}$, zigzag-like for $r>r_{\mathrm{cr}}$ ) for $N=60$. Degeneracy of all vibrational modes is observed at $r_{\mathrm{d}}$. The inset depicts how $r_{\mathrm{cr}}$ and $r_{\mathrm{d}}$ converge to a common value $r_{\infty}$ in the thermodynamic limit.

manifolds is very complex allowing for a large number of stationary states for given parameters $[8,9]$.

Here, we focus on the low-energy crystalline configurations and their equilibrium properties for an even number of particles $N$ which divides the number of windings $M$, i.e., $M=n N$ with $n=1,2, \ldots$ and $v=1 / n \leqslant 1$ being the filling factor. Then, the polygonic configuration $u_{j}^{(0)}=2(j-1) \pi n$ of the ring persists as a (stable or unstable) equilibrium configuration for all values of $r$ with the charges being located equidistantly, $\Delta_{j}^{(0)}=2 \pi n$, at the outer circle of the toroidal helix [Fig. 1(a)]. However, for sufficiently large $N$ (e.g., $N>4$ for $v=\frac{1}{2}$ ) this configuration loses its stability at a finite $r=r_{\mathrm{cr}}$, undergoing a symmetry-breaking pitchfork bifurcation to a zigzag-like configuration [Fig. 1(b)] in which successive particles have alternatingly moved to positive and negative values of the $z$ coordinate of the vector $\mathbf{r}$ [Eq. (1)]. For a fixed filling, here $v=\frac{1}{2}$, the bifurcation point $r_{\mathrm{cr}}$ shifts to lower values of $r$ with increasing $N$ (thus also increasing $M$ ), tending to a finite value $r_{\infty}$ [Fig. 1(c), inset] in this thermodynamic limit, with a rather slow convergence rate. Surprisingly, it turns out that the value of $r_{\infty}$ depends only on the helix pitch $h$; namely, $r_{\infty}=\frac{h}{\sqrt{2} \pi}$ in physical units (or $r_{\infty}=\frac{1}{2 \sqrt{2}}$ in our dimensionless units), independently of $v$.

Following the stable branch of solutions, we calculate the spectrum of vibrational modes in the harmonic approximation [Fig. 1(c)]. Intriguingly, in the regime $r<r_{\text {cr }}$ (where the stable configuration is still symmetric) this spectrum exhibits a crossing point $r_{\mathrm{d}}$ where all modes are very close to degenerate. The value $r_{\mathrm{d}}$ also depends on the system size, decreasing for large $N$ and tending to $r_{\infty}$ in the thermodynamic limit [Fig. 1(c), inset], but much faster than $r_{\mathrm{cr}}$ does. Thus, for finite systems an interval $r_{\mathrm{d}}<r<r_{\text {cr }}$ always exists. In this region the spectrum is inverted, and finally the lowest eigenvalue crosses zero at $r_{\mathrm{cr}}$, rendering the symmetric configuration unstable and leading to the observed pitchfork bifurcation [Fig. 1(b)]. For $r>r_{\text {cr }}$ two branches of frequencies separated by a gap are created, as a result of the new emergent solutions possessing a doubled unit cell.

Vibrational analysis. We now return to the frequency spectrum of the symmetric, polygonic configuration for $r<$ $r_{d}$. This being a Wigner crystal with a one-particle unit cell, the corresponding dispersion relation consists of a single branch. For its evaluation, we introduce the arc length parametrization in which the kinetic energy and Euler-Lagrange equations assume the standard form [9]. The dispersion relation then reads

$$
\omega^{2}(k)=\frac{1}{a^{2}(R+r)^{2}+r^{2}} \sum_{l=1}^{N} H_{1, l} \exp [-i k(l-1) \Delta s],
$$

with the Hessian at the equilibrium configuration $H_{i, j}=$ $\left.\frac{\partial^{2} V}{\partial u_{i} \partial u_{j}}\right|_{\left\{u_{j}^{(0)}\right\}}$ (we can fix one index for symmetry reasons). The prefactor in Eq. (2) results from transforming to the arc length $s, \Delta s$ denotes the arc length interparticle distance of the symmetric solution, and $k=\frac{2 \pi m}{N \Delta s},\left(m=0, \pm 1, \ldots \pm \frac{N}{2}\right)$ is the wave number of the corresponding excitation.

Results for $\omega(k)$ at different values of $r$ are shown in Fig. 2. For $r=0$ [Fig. 2(b)] the long-wavelength limit $k \rightarrow 0$, corresponding to identical displacements of all particles (c.m. mode [Fig. 2(a)]) has a vanishing frequency $\omega \rightarrow 0$, resulting from the c.m. decoupling in the ring geometry.

As the helix radius $r$ increases, the spectrum at small $k$ becomes smoother and a gap opens at $k=0$ [Fig. 2(c)] due to the coupling of the c.m. to the relative motion for $r>0$. This gap increases with increasing $r$, while the overall width of the spectrum decreases. At a critical point $r_{\mathrm{d}}$ [Fig. 2(d)] the spectrum is essentially flat as we have already seen in Fig. 1(c). A zoom at this point [Fig. 2(d), inset] reveals that the degeneracy is very close to, but not complete. To locate the near-degeneracy point $r_{\mathrm{d}}$ analytically, we go back to Eq. (2). Complete degeneracy would imply that all offdiagonal elements of the Hessian $H_{i, j}, i \neq j$, vanish (the diagonal elements are always identical by symmetry). Focusing on the nearest-neighbor contributions, we thus find an approximate analytical expression for $r_{\mathrm{d}}$ by demanding $\left.H_{j, j+1}\right|_{r_{\mathrm{d}}}=0$, which yields

$$
r_{d}=a R \frac{\sqrt{3+\cos (2 a n \pi)}}{\sqrt{2}-a \sqrt{3+\cos (2 a n \pi)}},
$$




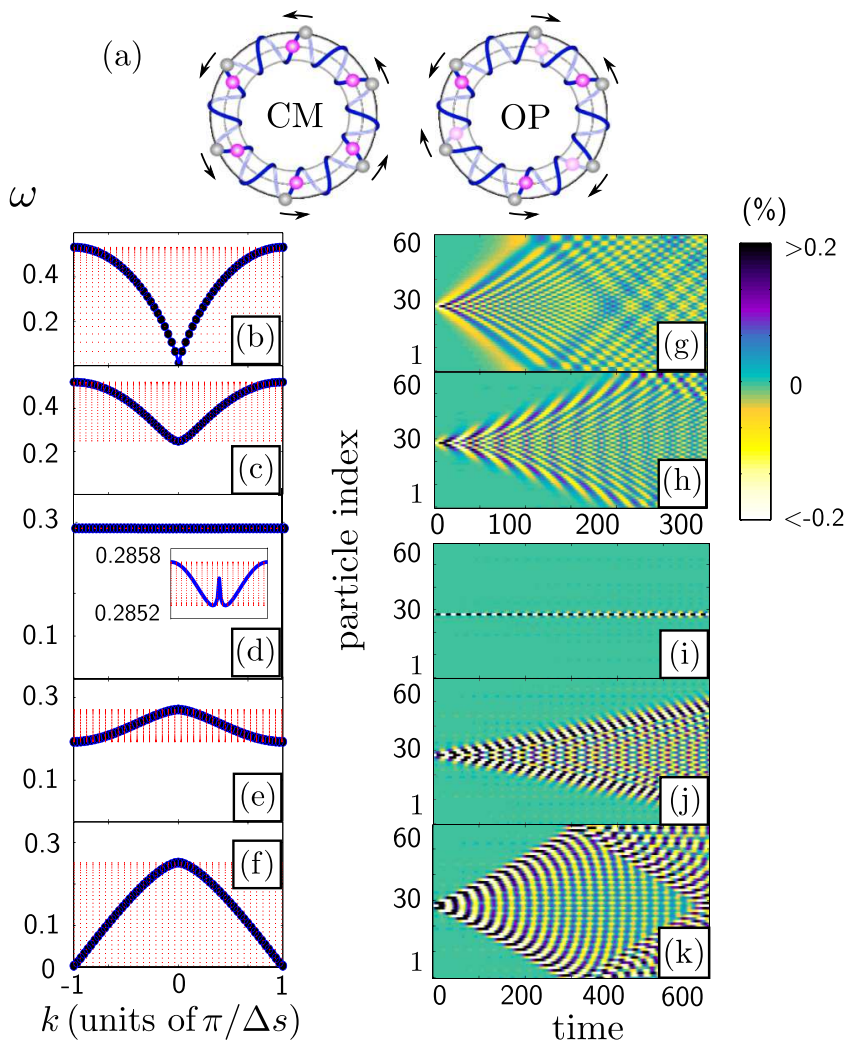

FIG. 2. (Color online) (a) Sketch of the center of mass (c.m., $k=0$ ) and the out-of-phase (OP, $k= \pm \frac{\pi}{\Delta s}$ ) modes. (b)-(f) Dispersion relation curves $\omega(k)$ for $N=60$ and increasing $r$ corresponding to the points $\left(1,2, r_{\mathrm{d}}, 3, r_{\mathrm{cr}}\right)$ marked in Fig. 1(c). For the same values of $r$, panels $(\mathrm{g})-(\mathrm{k})$ present the time evolution following an initial displacement of the particle at $j_{0}=29$ by $1 \%$ of $\Delta s$. Colors encode the displacement from equilibrium in units of $\Delta s$.

in excellent agreement with the numerical findings. Indeed, in the thermodynamic limit $R \rightarrow \infty, a \rightarrow 0, a R=\frac{1}{4}, r_{\mathrm{d}}$ tends to $r_{\infty}=\frac{1}{2 \sqrt{2}}$.

Beyond the crossing point, for $r>r_{\mathrm{d}}$, the curvature of the band changes sign permanently [Fig. 2(e)], implying that the OP mode [Fig. 2(a)] is now lower in frequency than the c.m. mode. The width of the spectrum increases again with increasing $r$ until at $r=r_{\text {cr }}$ the frequency of the OP mode at $k= \pm \pi / \Delta s$ reaches zero [Fig. 2(f)] and crosses to the imaginary axis for $r>r_{\mathrm{cr}}$, indicating the symmetric configuration becoming unstable due to the pitchfork bifurcation shown in Fig. 1(b). The condition $\left.\omega(k= \pm \pi / \Delta s)\right|_{r_{\mathrm{cr}}}=0$ can also be tackled analytically, giving an expression for $r_{\mathrm{cr}}$ which shows that it indeed tends to $r_{\infty}$ in the thermodynamic limit.

The almost full degeneracy of the linearization spectrum at $r_{\mathrm{d}}$ implies a remarkable localization property in the smallamplitude dynamics, illustrated in Figs. 2(g)-2(k). Here we explore the time evolution following a displacement of a single particle at site $j_{0}$ by $1 \%$ of $\Delta s$. Generically, this initially localized excitation spreads over the whole crystal; see, e.g., Fig. 2(g) for the case of a ring. More precisely, a cone structure emerges indicating a finite velocity at which the excitation proliferates into the crystal. This cone becomes narrower with decreasing bandwidth of the spectrum, see Fig. 2(h), until at
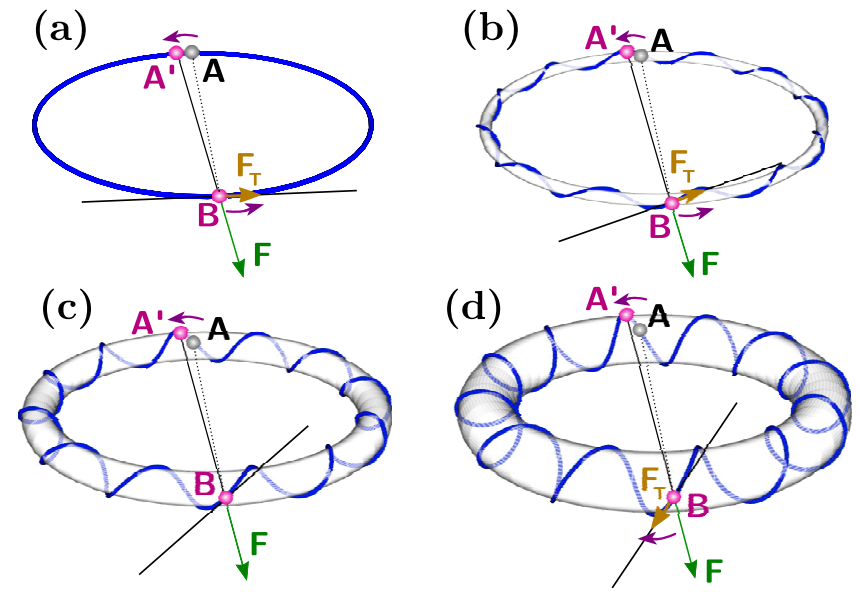

FIG. 3. (Color online) Schematic illustration of the response of a two-particle system $A, B$ to a single-particle displacement $A A^{\prime}$ for the cases: (a) $r=0$, (b) $r<r_{\mathrm{d}}$, (c) $r=r_{\mathrm{d}}$, and (d) $r>r_{\mathrm{d}}$. The total force $F$ acting on particle $B$ and its component $F_{T}$ tangential to the toroidal helix are shown, whereas curved arrows indicate the directions of displacements.

the point of near-degeneracy and thus near-zero bandwidth [Fig. 2(i)] the cone closes and the excitation no longer significantly spreads. This unique dynamical feature indicates the presence of an effective screening of interactions at $r=r_{\mathrm{d}}$, enabling essentially independent motion of the charged particles. We emphasize that, for this geometric configuration, any small initial excitation would maintain its shape for large times. Moving to larger radii $r>r_{\mathrm{d}}$, the degeneracy is lifted and the bandwidth of the spectrum increases again, thus reopening the cone [Figs. $2(\mathrm{j})$ and $2(\mathrm{k})]$.

Within the linearized equations, the initial dynamics of the spreading can be linked to $\omega(k)$ also on a formal level. The proliferation of the localized excitation can be quantified by the variance $S(t)=\sum_{j} j^{2} e_{j}(t)-j_{0}^{2}$, where we employ the local energy $e_{j}(t)$ at site $j$ as introduced in Ref. [13], with the time-independent normalization $\sum_{j} e_{j}=1$. Then similar arguments as in Ref. [14] apply, leading to $S(t) \propto t^{2} \int \mathrm{d} k\left|\frac{\mathrm{d} \omega}{\mathrm{d} k}\right|^{2}$, assuming the crystal is large enough to approximate a sum over $k$ with an integral over the first Brillouin zone. Consequently, the spreading of an initially localized excitation is ballistic, with a velocity determined by the square of the group velocity integrated over all $k$. If $\frac{\mathrm{d} \omega}{\mathrm{d} k}$ is close to zero globally, i.e., the dispersion is almost flat, $S(t)$ will grow only slowly with time and the excitation will spread only on very long timescales, which is what happens at $r_{\mathrm{d}}$.

Degeneracy point. We now provide a geometrical interpretation for the emergence of the degeneracy point $r_{d}$ in the spectrum. Let us examine the response of the simple $N=2$ particle equilibrium configuration to a single-particle displacement (Fig. 3). A slight counterclockwise displacement of particle $A$ towards the position $A^{\prime}$ results in a force acting on particle $B$. For the cases $r=0, r<r_{\mathrm{d}}$ [Figs. 3(a) and 3(b)], this force possesses a component tangential to the confining manifold, causing a counterclockwise acceleration of particle $B$. At $r_{\mathrm{d}}$ [Fig. 3(c)], the geometry is such that the displacement $A A^{\prime}$ results in a force that has no component tangential to the toroidal helix curve at the equilibrium position of $B$ and 
is therefore entirely compensated by the constraint. Thus, the small-amplitude motion of particle $B$ is effectively decoupled from that of $A$. This simple geometric condition indeed leads to the same value of $r_{\mathrm{d}}$ as Eq. (3) for $N=2$. For $r>r_{\mathrm{d}}$ the force acting on $B$ again attains a nonvanishing projection onto the tangential but is now oriented in the opposite direction, causing a clockwise acceleration of particle $B$, in line with the observed inversion of the dispersion relations in this regime [Figs. 2(e) and 2(f)]. For $N>2$, the geometry parameters can no longer be chosen such that all forces acting on the other particles after displacing a particular one are strictly compensated by the constraint. Still, it can be seen that the tangential projection of the force acting on particle $j$ after particle $i$ has been slightly displaced is proportional to the Hessian matrix element $H_{i, j}$. We have seen above that at $r_{\mathrm{d}}$ all these $H_{i, j} \approx 0$ for $i \neq j$. Thus, at the point of degeneracy, the geometry is such that if a single particle is displaced, all resulting force projections onto the local tangents where the other particles sit are very small simultaneously, resulting in the effective decoupling.

Conclusions. We have shown that, for charged particles confined on a 1D closed helix, a linear to zigzag-like bifurcation occurs when increasing the radius of the helix at commensurate fillings. Similar transitions are known for linear Wigner crystals under harmonic [15-20] or octupole trapping $[21,22]$, where they are driven by the increment of dimensionality from one dimension to three dimensions controlled by the transverse trapping potential. In our case, however, the single-particle configuration space remains always strictly $1 \mathrm{D}$, which manifests itself in the way in which the critical value $r_{\text {cr }}$ is reached. In particular, for the transition to occur, the OP mode (ultimately causing the symmetry-breaking deformation of the crystal) has to cross zero at $r_{\mathrm{cr}}$, in contrast to the ring limit $r=0$ where the OP mode has the largest frequency. This necessarily implies an inversion of the dispersion relation when approaching $r_{\mathrm{cr}}$, since the reduced dimensionality precludes a transverse branch which usually causes the bifurcation $[17,18]$. Notably, the deformation of the dispersion curve when increasing $r$ towards $r_{\mathrm{cr}}$ passes through a point where all modes are essentially degenerate and the dispersion is flat. For this particular geometry, the (small-amplitude) dynamics of the particles is effectively decoupled, allowing for localized, nonspreading excitations.

The present setup offers unprecedented control over the vibrational band structure, suggesting its potential usefulness for applications in electronic nanodevices such as free-standing helix tubules [23-25]. The possibility of geometrically localizing excitations may be of interest for the design of acoustic meta-materials [26] and for applications in phononics such as sound isolation and cloaking [27] or even information storage [28]. Beyond this, helical structures are abundantly found in organic or inorganic molecules [29,30], and recent advances in optical trapping of ions [31] and helical trap design for neutral atoms [32-34] may pave the way for future experimental realizations with ultracold ions.

Acknowledgments. A.V.Z. thanks C. Morfonios and P. G. Kevrekidis for fruitful discussions and acknowledges support through a Ph.D. scholarship from the International Max Planck Research School for Ultrafast Imaging and Structural Dynamics (IMPRS-UFAST). J.S. gratefully acknowledges support from the Studienstiftung des deutschen Volkes.
[1] D. H. E. Dubin and T. M. O’Neil, Rev. Mod. Phys. 71, 87 (1999).

[2] T. Lahaye, C. Menotti, L. Santos, M. Lewenstein, and T. Pfau, Rep. Prog. Phys. 72, 126401 (2009).

[3] R. H. French et al., Rev. Mod. Phys. 82, 1887 (2010).

[4] M. Boninsegni and N. V. Prokof'ev, Rev. Mod. Phys. 84, 759 (2012).

[5] O. V. Kibis, Phys. Lett. A 166, 393 (1992).

[6] O. V. Kibis et al., Electromagn. 25, 425 (2005).

[7] K. T. Law and D. E. Feldman, Phys. Rev. Lett. 101, 096401 (2008).

[8] P. Schmelcher, Europhys. Lett. 95, 50005 (2011).

[9] A. V. Zampetaki, J. Stockhofe, S. Krönke, and P. Schmelcher, Phys. Rev. E 88, 043202 (2013).

[10] J. K. Pedersen, D. V. Fedorov, A. S. Jensen, and N. T. Zinner, J. Phys. B: At., Mol. Opt. Phys. 47, 165103 (2014).

[11] E. Wigner, Phys. Rev. 46, 1002 (1934).

[12] R. C. Thomson, arXiv:1411.4945v1.

[13] P. B. Allen and J. Kelner, Am. J. Phys. 66, 497 (1998).

[14] A. J. Martínez and M. I. Molina, J. Phys. A: Math. Theor. 45, 275204 (2012).

[15] R. W. Hasse and J. P. Schiffer, Ann. Phys. (NY) 203, 419 (1990).

[16] G. Birkl, S. Kassner, and H. Walther, Nature (London) 357, 310 (1992).
[17] G. Piacente, I. V. Schweigert, J. J. Betouras, and F. M. Peeters, Phys. Rev. B 69, 045324 (2004).

[18] S. Fishman, G. DeChiara, T. Calarco, and G. Morigi, Phys. Rev. B 77, 064111 (2008).

[19] E. Shimshoni, G. Morigi, and S. Fishman, Phys. Rev. Lett. 106, 010401 (2011).

[20] A. C. Mehta, C. J. Umrigar, J. S. Meyer, and H. U. Baranger, Phys. Rev. Lett. 110, 246802 (2013).

[21] E. Yurtsever, E. D. Onal, and F. Calvo, Phys. Rev. A 83, 053427 (2011).

[22] F. Cartarius, C. Cormick, and G. Morigi, Phys. Rev. A 87, 013425 (2013).

[23] V. Y. Prinz et al., Phys. E (Amsterdam, Neth.) 6, 828 (2000).

[24] O. G. Schmidt and K. Eberl, Nature (London) 410, 168 (2001).

[25] Nanotubes and Nanofibers edited by Y. Gogotsi (Taylor \& Francis Group, LLC New York, 2006).

[26] P. A. Deymier, Acoustic Metamaterials and Phononic Crystals, Springer Series in Solid-State Sciences (Springer, Berlin, 2013).

[27] M. Maldovan, Nature (London) 503, 209 (2013).

[28] T. J. G. Apollaro and F. Plastina, Open Sys. Info. Dyn. 14, 41 (2007).

[29] A. A. Kornyshev, D. J. Lee, S. Leikin, and A. Wynveen, Rev. Mod. Phys. 79, 943 (2007). 
[30] Y. Wang, J. Xu, Y. Wang, and H. Chen, Chem. Soc. Rev. 42, 2930 (2013).

[31] Ch. Schneider, M. Enderlein, T. Huber, and T. Schaetz, Nat. Photonics 4, 772 (2010).

[32] A. Okulov, Phys. Lett. A 376, 650 (2012).
[33] E. Vetsch, D. Reitz, G. Sagué, R. Schmidt, S. T. Dawkins, and A. Rauschenbeutel, Phys. Rev. Lett. 104, 203603 (2010).

[34] D. Reitz and A. Rauschenbeutel, Opt. Commun. 285, 4705 (2012). 